\title{
Mechanical, dynamic, and thermomechanical properties of coir/pineapple leaf fiber reinforced polylactic acid hybrid biocomposites
}

\begin{abstract}
Natural fiber-based polymer composites have been widely studied to substitute synthetic materials. In this research, pineapple leaf fibers (PALF) and coir fibers (CF) were loaded into a polylactic acid (PLA) matrix to develop composite materials with improved mechanical and thermal properties, which could be potentially applied as biodegradable food packaging. Biocomposites with different fiber ratios were manufactured using an internal mixer plasticizer and a hot press machine. Mechanical and thermal analyses of the obtained composites were carried out and the results were compared with those of pure PLA. Scanning electron microscopy (SEM) was used to observe the microstructural failure of the composites. Mechanical tests indicated that all the composites had higher tensile and flexural modulus, compared to those of neat PLA. Also, strength values were increased upon addition of PALF, while impact tests showed enhanced strength results upon addition of CF. SEM findings confirmed the outcomes of the mechanical tests. DMA results confirmed that the storage and loss moduli of the CF/PALF/PLA hybrid composites increased with respect to those of the neat PLA, whereas the $\tan \delta$ decreased. The coefficient of thermal expansion (CTE) of the PLA composites decreased with the addition of fiber reinforcements. Based on the results achieved in this investigation, the hybrid composite containing CF and PALF in a 1:1 ratio (C1P1) presented the optimum set of mechanical properties and improved thermal stability, which make it suitable for applications such as food packaging and structure components to help reduce the environmental loads.
\end{abstract}

